\title{
ANÁLISE CIENCIOMÉTRICA DA ORDEM RODENTIA (MAMMALIA: ERETHIZONTIDAE) COMO FERRAMENTA PARA O DELINEAMENTO DE ÁREAS PRIORITÁRIAS À CONSERVAÇÃO
}

\section{SCIENTOMETRIC ANALYSIS OF THE ORDER RODENTIA (MAMMALIA: ERETHIZONTIDAE) AS A TOOL FOR THE DELINEATION OF PRIORITY AREAS FOR CONSERVATION}

\author{
Rodrigo Leite Arrieira ${ }^{1}$; Thaís Fernandes Mendonça Mota² $^{2}$; Henrique Ortêncio Filho ${ }^{3}$ \\ Universidade Estadual de Maringá, Departamento de Biologia. Avenida Colombo, n5790, \\ Zona 7, Maringá-PR, CEP: 87020-900. E-mail: rodrigoarrieira@yahoo.com.br \\ ${ }^{1}$ Pós-graduando em Biologia Comparada, Universidade Estadual de Maringá. Endereço para \\ correspondência: Rua Maísa nº401, CEP 87053-060, Conjunto Cidade Alta, Maringá-PR. \\ E-mail: rodrigoarrieira@yahoo.com.br. Fone: (44) 9903-0021. \\ 2 Pós-graduanda em Biologia Comparada, Universidade Estadual de Maringá. E-mail: \\ tfmm_0412@hotmail.com \\ 3 Professor do Departamento de Ciências, Universidade Estadual de Maringá. E-mail: \\ henfilhobat@gmail.com
}

Data de recebimento: 20/09/2013

Data da aprovação: 15/10/2013

\section{RESUMO}

Rodentia é a ordem de mamíferos que apresenta o maior número de espécies, representando $42 \%$ da biodiversidade mundial. No Brasil ocorrem 75 gêneros e 243 espécies que apresentam grande diversidade em sua morfologia, comportamento, modo de vida e distribuição. São considerados bons indicadores ambientais e o conjunto de informações a respeito desse grupo é fundamental para o delineamento de áreas destinadas à conservação. Entre os métodos aplicados para a avaliação da ciência, as análises cienciométricas permitem avaliar a produção científica em uma determinada área. Este trabalho objetivou realizar um estudo quantitativo da produção científica sobre a Ordem Rodentia no Brasil e, mais especificamente, no estado do Paraná, entre os anos de 2003 e 2012. O levantamento bibliográfico foi realizado por meio do banco de dados publicados no sítio do "Thomson Reuters Web of Knowledge", entre 2003 e 2012, e os trabalhos selecionados foram agrupados de acordo com o ano da publicação, bioma, temas e espécies pesquisadas. Foram identificados quatro artigos do estado do Paraná e 154 artigos do Brasil. O ambiente com maior número de publicações foi a Mata Atlântica. A maior parte das pesquisas foi desenvolvida na área de genética, com $31,8 \%$. As principais espécies pesquisadas no período foram Akodon montensis, Akodon cursor e Oligoryzomys nigripes. O levantamento realizado por este trabalho contribui para o conhecimento do estado da arte do grupo e destaca a necessidade de se ampliarem os estudos onde esta ordem ainda não é estudada, para o melhor delineamento de áreas prioritárias à conservação.

Palavras-chave: Roedores. Biomas. Biodiversidade. Pequenos mamíferos. Cienciometria.

\footnotetext{
ABSTRACT

Rodentia is the order of mammals that has the highest number of species representing $42 \%$ of global biodiversity. In Brazil there are 75 genera and 243 species that are diverse 
in their morphology, behavior, lifestyle and distribution. They are considered good environmental indicators and other data about this group is critical for the design of areas to conservation. Among the methods used for the evaluation of science, scientometric analyzes allows to assess the scientific literature in a given area. This study aimed to perform a quantitative study of the scientific literature on the Order Rodentia in Brazil and, more specifically, in Paraná State, between the years 2003 and 2012. The literature review was performed using the database published on the site "Thomson Reuters Web of Knowledge", between 2003 and 2012, and selected studies were grouped according to the year of publication, biome, themes and species surveyed. We identified four articles from Paraná and 154 articles of Brazil. The environment with the highest number of publications was the Atlantic forest. Most of the research has been developed in the genetic field with $31.8 \%$. The main species surveyed in the period were Akodon montensis, Akodon cursor and Oligoryzomys nigripes. The survey conducted for this work contributes to the knowledge of the state of the art group and highlights the need to expand the studies where this order is not yet studied, contributing to a better delineation of areas for conservation.

Keywords: Rodents. Biomes. Biodiversity. Small mammals. Scientometry.

\section{Introdução}

A diversidade de mamíferos no Brasil é considerada uma das maiores do mundo (REIS, 2011). Entre as ordens de mamíferos, a Ordem Rodentia é a que apresenta o maior número de espécies, aproximadamente $42 \%$ da biodiversidade de mamíferos de todo o mundo (WILSON; REEDER, 2005). No Brasil, ocorrem 75 gêneros e 243 espécies, que apresentam grande diversidade em sua morfologia, comportamento, modo de vida e distribuição. Seus gêneros são caracterizados de acordo com as dimensões externas e o tipo e coloração dos pelos. São incluídos neste grupo os ratos, preás, capivaras, pacas e cutias (REIS et al., 2011).

Os representantes dessa ordem, em geral, possuem tamanho pequeno, são versáteis, esguios e com adaptações que favorecem variados modo de vida, como arborícola, terrestre, semiaquático ou fossorial. Essa variedade de adaptações permite que os roedores sejam encontrados em diferentes climas e tipos de vegetação (EMMONS; FEER, 1997). Além disso, o sucesso adaptativo do grupo é atribuído a algumas características como: elevada taxa reprodutiva, comportamento ágil, hábito alimentar diversificado e tamanho corporal reduzido na maioria. Contudo, a característica mais marcante do grupo é a dentição, composta por um par de incisivos superiores grandes e de crescimento contínuo (SMITH; PATTON, 1999; NOWAK, 1999).

Nos últimos anos, tornou-se crescente a preocupação com o monitoramento da produção científica e, para realizar essa avaliação, estudos métricos, quantitativos ou qualitativos, são cada vez mais necessários. Entre os métodos aplicados para avaliação da ciência, destaca-se a cienciometria, que pode ser definida como a área voltada aos estudos quantitativos das atividades científicas, relacionados à produção, propagação e utilização de informações científicas, por um determinado país, comunidade científica, ou instituição (SPINAK, 1998).

Considerando a elevada diversidade desses mamíferos no Brasil, tendo em vista a importância e carência de estudos sobre os roedores, o objetivo do presente trabalho foi realizar um estudo quantitativo da produção científica sobre a Ordem Rodentia no estado do Paraná e no Brasil, entre os anos de 2003 e 2012.

\section{Material e método}

O levantamento dos estudos foi realizado por meio do banco de dados publicados no sítio do Thomson Reuters Web of Knowledge, durante o período de 2003 a 2012. Foram realizados dois tipos de investigação: uma, utilizando os descritores de busca ordenando, na primeira linha, o termo "Rodentia", e na segunda linha o termo "Paraná", 
visando agrupar os artigos indexados desenvolvidos com a Ordem Rodentia (Mammallia) no estado do Paraná, Brasil. A outra investigação abordou a busca combinada dos descritores: "Rodentia" (primeira linha) e "Brazil"(segunda linha), objetivando abranger as pesquisas publicadas em todo o Brasil sobre esta ordem.

Os trabalhos selecionados foram agrupados de acordo com o ano da publicação, bioma, a área de estudo da pesquisa e espécies citadas no artigo.

\section{Resultados e discussão}

\section{Trabalhos realizados no estado do Paraná e no Brasil}

Pela ordenação da pesquisa com os termos "Rodentia" e "Paraná", foram identificados apenas quatro artigos, no período de 2003 a 2012, realizados com essa Ordem de mamíferos no estado do Paraná (Tabela 1).

Tabela 1 - Artigos realizados no estado do Paraná no período pesquisado.

\begin{tabular}{|c|c|c|c|}
\hline Título & Autores e ano & Tema & Instituição \\
\hline $\begin{array}{l}\text { Diet of Sciurus ingrami Thomas (Rodentia, Sciuridae) on an } \\
\text { Araucauria Pine Forest remnant, Parana, Brazil. }\end{array}$ & $\begin{array}{l}\text { MIRANDA, J. M. D. et al., } \\
2005\end{array}$ & Forrageamento & UFPR \\
\hline $\begin{array}{l}\text { First genotoxicity study of Parana river water from Argentina using } \\
\text { cells from the clam Corbicula fluminea (Veneroida Corbiculidae) and } \\
\text { Chinese hamster (Cricetulus griseus Rodentia, Cricetidae) K1 cells in } \\
\text { the comet assay. }\end{array}$ & $\begin{array}{l}\text { CAFETTI, J. D. et al., } \\
2008\end{array}$ & Genética & UNaM e UEL \\
\hline $\begin{array}{l}\text { Geographic distribution of small non-volant mammals in the } \\
\text { Araguaia and Parana basins, south-central region of Brazil. }\end{array}$ & $\begin{array}{l}\text { CACERES, N. C. et al., } \\
2008\end{array}$ & $\begin{array}{l}\text { Distribuição de } \\
\text { espécies }\end{array}$ & $\begin{array}{l}\text { UFMS, INPA e } \\
\text { UFSM }\end{array}$ \\
\hline $\begin{array}{l}\text { Cytogenetic characterization of Metynnis maculatus (Teleostei; } \\
\text { Characiformes): the description in Serrasalminae of a small B } \\
\text { chromosome bearing inactive NOR-like sequences. }\end{array}$ & SABRINA, B. et al., 2009 & Genética & USP \\
\hline
\end{tabular}

Já a busca combinada de publicações em todo o Brasil relacionadas ao grupo, com os termos "Rodentia" e "Brazil", foram selecionados 154 artigos científicos publicados no período pesquisado. Essa quantidade de publicações pode ser considerada reduzida, por se tratar da ordem com maior diversidade de espécie dentre os mamíferos, com 75 gêneros e 243 espécies brasileiras registradas (REIS et al., 2011). Além disso, é inferior quando comparada com a quantidade de trabalhos realizados com outras ordens de mamíferos, como, por exemplo, a Ordem Chiroptera, segunda maior entre os mamíferos em riqueza de espécies, com 172 espécies identificadas no Brasil. (REIS et al, 2011). Sobre esta ordem foi obtido um maior número de trabalhos desenvolvidos no país, a saber, 192 artigos encontrados, quando pesquisados a partir dos mesmos critérios e descritores utilizados para o grupo Rodentia.

Essa baixa produtividade científica sobre a Ordem Rodentia, tanto no estado do Paraná quanto em todo o Brasil, reflete a carência de conhecimento acerca desses roedores. Por serem considerados bons indicadores ambientais, devido à sua grande capacidade de adaptação, o conjunto de informações a respeito desse grupo é fundamental para o delineamento de áreas destinadas à conservação ou na detecção de áreas que sofrem com o elevado impacto antrópico (TIEPOLO, 2013).

\section{Trabalhos realizados nos biomas brasileiros}

De acordo com o Ministério do Meio Ambiente (2002), no Brasil, os ambientes terrestres são classificados em seis biomas: Mata Atlântica, Pampas ou Campos Sulinos, Cerrado, Pantanal, Caatinga e Amazônia. Os artigos foram caracterizados de acordo com o bioma estudado no trabalho. O ambiente com maior número de publicações foi a Mata Atlântica, com 77 artigos, correspondendo à metade dos trabalhos selecionados por essa pesquisa, conforme apresentado na Tabela 2. 
Tabela 2 - Trabalhos envolvendo a ordem Rodentia publicados no Brasil, de acordo com o bioma registrado e ano.

\begin{tabular}{lcccccccccc}
\hline & 2003 & 2004 & 2005 & 2006 & 2007 & 2008 & 2009 & 2010 & 2011 & 2012 \\
\hline Caatinga & 0 & 1 & 0 & 0 & 1 & 2 & 0 & 0 & 1 & 1 \\
Cerrado & 2 & 3 & 2 & 0 & 3 & 0 & 0 & 1 & 5 & 4 \\
Floresta Amazônica & 0 & 2 & 1 & 0 & 2 & 1 & 0 & 0 & 0 & 3 \\
Mata Atlântica & 4 & 3 & 4 & 3 & 7 & 9 & 8 & 12 & 17 & 10 \\
Pampa & 1 & 2 & 1 & 4 & 4 & 0 & 3 & 4 & 1 & 4 \\
Pantanal & 0 & 1 & 0 & 0 & 0 & 0 & 0 & 0 & 0 & 0 \\
Diversos & 0 & 1 & 1 & 1 & 3 & 1 & 2 & 2 & 3 & 3
\end{tabular}

O maior número de publicações constatadas no bioma Mata Atlântica deve-se, principalmente, à sua maior diversidade de espécies e endemismo (RIBEIRO et al., 2009). Esse ambiente, originalmente, estendia-se do Rio Grande do Norte ao Rio Grande do Sul, no Brasil, além do oeste do Paraguai e norte da Argentina, e atualmente está reduzido a menos de $7 \%$ a $10 \%$ de sua distribuição original no Brasil (BRITO, 2004; METZGER, 2009).

\section{Temas pesquisados nos trabalhos}

Considerando o número total de artigos e a área de estudo em que foi desenvolvida a pesquisa, observou-se que, do total de 154 artigos, a maior parte foi desenvolvida na área de genética, com $31,8 \%$, seguida de parasitologia e registro de novas espécies, com $17,5 \%$ e $9,1 \%$, respectivamente (Figura 1 ).

Figura 1 - Trabalhos publicados envolvendo a ordem Rodentia, de acordo com a área de pesquisa.

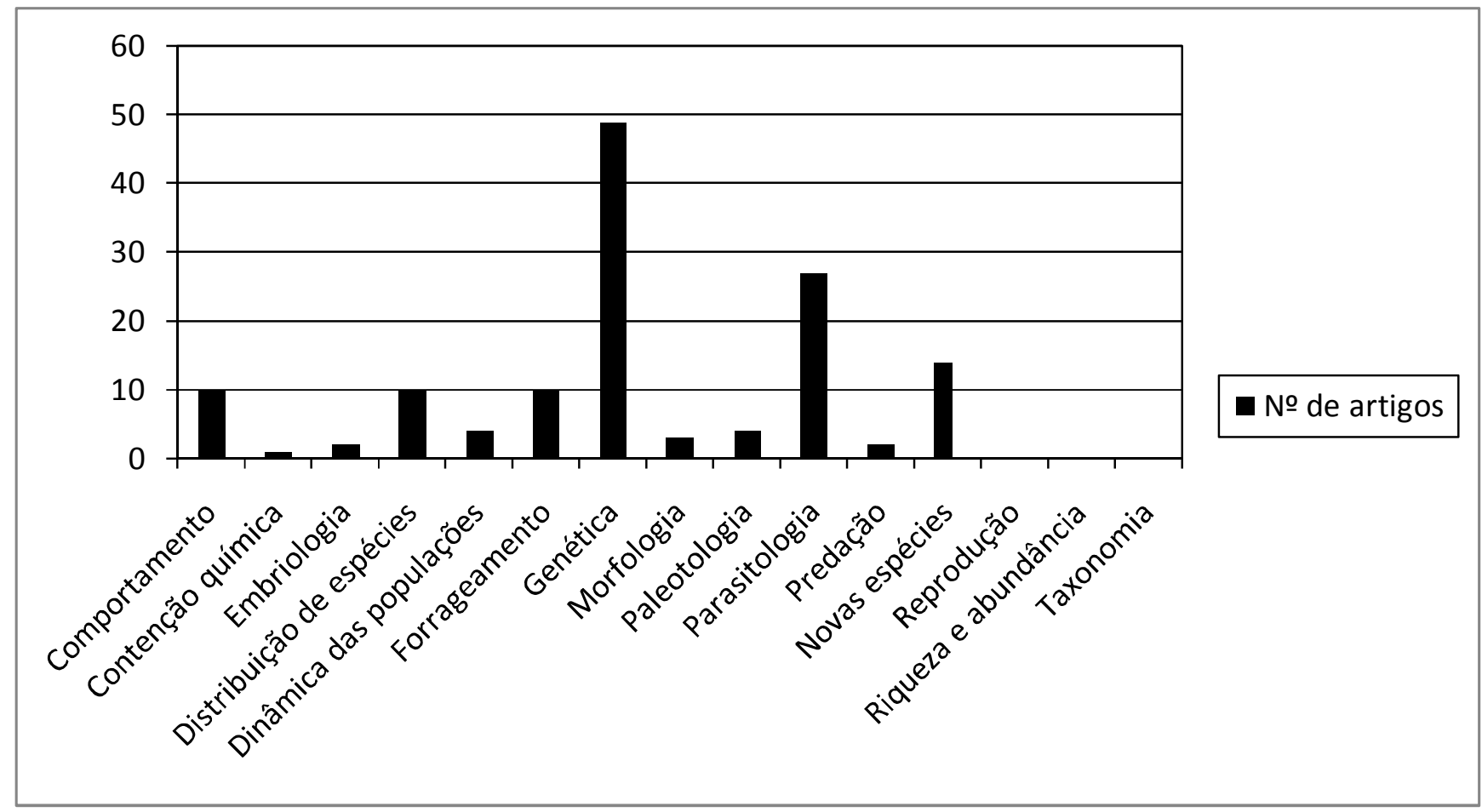

A grande contribuição dos temas publicados na área de genética, num total de 49 artigos, deve-se, principalmente, à existência de gêneros de difícil identificação taxonômica, considerando-se que somente as características morfológicas não são suficientes para a diferenciação em espécies, ocorrendo a necessidade de realização de cariotipagem e posterior comparação com dados morfológicos para uma correta classificação do exemplar (PATTERSON, 2002). 
Já os diversos artigos publicados com os temas em parasitologia refletem a ampla importância sanitária do grupo, como possíveis reservatórios de organismos causadores de doenças, tais como Tripanomossoma cruzi (doença de Chagas) (HERRERA et al., 2004), Schistosoma mansoni (esquistossomose) (MALDONADO et al., 2006), Leptospira spp. (leptospirose) (ULLMANN et al., 2012) e Paracoccidioides brasiliensis (paracoccidioidomicose) (BERBERT et al., 2007), e sua importância veterinária por serem parasitados por vermes helmínticos (GONÇALVES et al., 2007), ácaros (SARAIVA et al., 2012) e protozoários (LAINSON et al., 2007).

Pôde-se observar um grande número de publicações em que foram registradas novas espécies por meio de revisões sistemáticas ou pesquisa em novas áreas de estudo, caracterizando a morfologia, biologia e comportamento dos novos indivíduos abordados (Tabela 3). Esses estudos reforçam a necessidade de ampliar as pesquisas no Brasil, visando conhecer e preservar a sua biodiversidade (PINHEIRO et al., 2004).

Tabela 3 - Artigos envolvendo a ordem Rodentia, com registro de novas espécies e ano de publicação.

\begin{tabular}{|c|c|}
\hline Título & Ano \\
\hline A new species of Oryzomys (Rodentia, Sigmodontinae) of the subflavus group from the Cerrado of Central Brazil. & 2003 \\
\hline $\begin{array}{l}\text { New record of Rhagomys rufescens (Thomas 1886) (Rodentia: Muridae: Sigmodontinae) in the Atlantic forest of } \\
\text { southeastern Brazil. }\end{array}$ & 2004 \\
\hline A new species of Wiedomys (Rodentia: Sigmodontinae) from Brazilian Cerrado. & 2005 \\
\hline A new species of Juliomys (Mammalia: Rodentia: Cricetidae) from the Atlantic forest of southeastern Brazil. & 2007 \\
\hline New records of Rhagomys rufescens (Rodentia: Sigmodontinae) in the Atlantic forest of Brazil. & 2008 \\
\hline $\begin{array}{l}\text { Systematic review of genus Cerradomys Weksler, Percequillo and Voss, } 2006 \text { (Rodentia: Cricetidae: Sigmodontinae: } \\
\text { Oryzomyini), with description of two new species from eastern Brazil. }\end{array}$ & 2008 \\
\hline A New Species of Atlantic Forest Tree Rat, Genus Phyllomys (Rodentia, Echimyidae) from Southern Brazil. & 2008 \\
\hline Potential distribution and new records of Trinomys species (Rodentia: Echimyidae) in the state of Rio de Janeiro. & 2009 \\
\hline A new species of Calomys (Rodentia: Sigmodontinae) from Eastern Brazil. & 2010 \\
\hline $\begin{array}{l}\text { Phylogeography of Rhipidomys (Rodentia: Cricetidae: Sigmodontinae) and description of two new species from southeastern } \\
\text { Brazil. }\end{array}$ & 2011 \\
\hline New species of Cerradomys from coastal sandy plains of southeastern Brazil (Cricetidae: Sigmodontinae). & 2011 \\
\hline Small mammals of the mid-Araguaia River in central Brazil, with the description of a new species of climbing rat. & 2011 \\
\hline $\begin{array}{l}\text { A new genus and species of rodent from the Brazilian Atlantic Forest (Rodentia: Cricetidae: Sigmodontinae: Oryzomyini), } \\
\text { with comments on Oryzomyine biogeography. }\end{array}$ & 2011 \\
\hline $\begin{array}{l}\text { An endemic new species of tuco-tuco, genus Ctenomys (Rodentia: Ctenomyidae), with a restricted geographic distribution } \\
\text { in southern Brazil. }\end{array}$ & 2012 \\
\hline
\end{tabular}

Os diversos artigos encontrados com os temas em ecologia, nas áreas de comportamento, distribuição de espécies, dinâmica de populações, forrageamento, predação, reprodução e riqueza e abundância de espécies, refletem a importância dos estudos ecológicos de roedores no Brasil. Esses trabalhos, tais como publicações sobre a distribuição das espécies, caracterizam a composição de espécies no ambiente, contribuindo para a tomada de medidas para a regeneração de áreas impactadas e delineamento de áreas destinadas ao manejo e conservação, além de estabelecimento de parâmetros para comparação com áreas degradadas ou fragmentadas (PARDINI; UMETSU, 2006).
A contenção química foi o tema menos abordado, com apenas um artigo, apesar de estudos dessa natureza serem relevantes, uma vez que os aspectos éticos devem ser ponto de discussão na pesquisa envolvendo a fauna, de maneira geral, além de garantir a segurança do animal e do pesquisador (STEIN et al., 2009).

Outro apontamento sobre os artigos selecionados é que vários tratam da Ordem Rodentia como parte de estudos desenvolvidos com pequenos mamíferos ou mamíferos não voadores. Comumente, os trabalhos são realizados agrupando esses animais pelo fato do tamanho corporal, hábitos e, consequentemente, métodos de captura 
para vários mamíferos e pequenos roedores brasileiros serem similares, favorecendo o estudo ecológico em conjunto (PREVEDELLO et al., 2008).

\section{Espécies citadas nos trabalhos}

Nos trabalhos selecionados foram citados 47 gêneros e 113 espécies, dentre os 75 gêneros e as 243 espécies existentes (REIS et al., 2011). Apesar de escassos, os trabalhos realizados no país para o grupo, as pesquisas abordaram uma quantidade significativa de táxons e os citam diversas vezes nos artigos selecionados (Tabela 4). As principais espécies pesquisadas no período foram Akodon montensis (Figura 2a) e Akodon cursor (Figura 2b), citadas em nove artigos, e Oligoryzomys nigripes (Figura 2c) citada em sete artigos. Estas três espécies são endêmicas do Brasil, podendo ser restritas ao bioma Mata Atlântica (BONVICINO, 2008).

Tabela 4 - Número de citações para cada espécie abordada nos trabalhos selecionados.

\begin{tabular}{|c|c|c|c|}
\hline Família & Gênero & Espécie & Quantidade de citações \\
\hline \multirow[t]{8}{*}{ Caviidae } & Cavia & Cavia intermedia & 1 \\
\hline & & Cavia magna & 1 \\
\hline & & Cavia aperea & 2 \\
\hline & Galea & Galea meyen & 1 \\
\hline & & Galea spixii & 1 \\
\hline & Hydrochaeris & Hydrochaeris hydrochaeris & 1 \\
\hline & & Hydrochoerus hydrochaeris & 2 \\
\hline & Kerodon & Kerodon rupestres & 1 \\
\hline \multirow[t]{24}{*}{ Cricetidae } & Abrawayaomys & & 1 \\
\hline & Akodon & Akodon lindberghi & 1 \\
\hline & & Akodon montensis & 9 \\
\hline & & Akodon paranaensis & 3 \\
\hline & & Akodon $\mathrm{sp}$ & 1 \\
\hline & & Akodon cursor & 9 \\
\hline & & Akodon serrensis & 1 \\
\hline & Blarinomys & Blarinomys breviceps & 1 \\
\hline & Bolomys & Bolomys lasiurus & 2 \\
\hline & Brazilian & Brazilian akodontines & 1 \\
\hline & Brucepattersonius & Brucepattersonius albinasus & 1 \\
\hline & & Brucepattersonius iheringi & 1 \\
\hline & & Brucepattersonius soricinus & 1 \\
\hline & Calomys & & 1 \\
\hline & & Calomys tocantinsi & 4 \\
\hline & & Calomys cerqueirai & 1 \\
\hline & & Calomys tener & 1 \\
\hline & & Calomys callosus & 1 \\
\hline & Cerradomys & Cerradomys goytaca & 1 \\
\hline & & Cerradomys langguthi & 1 \\
\hline & Delomys & Delomys dorsalis & 1 \\
\hline & & Deltamys kempi & 1 \\
\hline & Drymoreomys & Drymoreomys albimaculatus & 1 \\
\hline & Euryoryzomys & Euryoryzomys sp & 1 \\
\hline
\end{tabular}


continuação

\begin{tabular}{|c|c|c|c|}
\hline Família & Gênero & Espécie & Quantidade de citações \\
\hline & & Euryoryzomys russatus & 1 \\
\hline & Holochilus & Holochilus sciureus & 1 \\
\hline & Hylaeamys & Hylaeamys megacephalus & 1 \\
\hline & Juliomys & & 1 \\
\hline & & Juliomys pictipes & 1 \\
\hline & & Juliomys rimofrons & 1 \\
\hline & & Juliomys ossitenuis & 2 \\
\hline & Kunsia & Kunsia tomentosus & 1 \\
\hline & Necromys & Necromys lasiurus & 4 \\
\hline & & Nectomys squamipes & 4 \\
\hline & Oecomys & Oecomys catherinae & 1 \\
\hline & & Oecomys paricola & 1 \\
\hline & & Oecomys cleberi & 1 \\
\hline & & Oecomys bicolor & 1 \\
\hline & & Oecomys roberti & 1 \\
\hline & & Oecomys mamorae & 1 \\
\hline & Oligoryzomys & & 1 \\
\hline & & Oligoryzomys cf. microtis & 2 \\
\hline & & Oligoryzomys flavescens & 2 \\
\hline & & Oligoryzomys nigripes & 7 \\
\hline & Oryzomyini & & 2 \\
\hline & Oryzomys & & 1 \\
\hline & & Oryzomys scotti & 1 \\
\hline & & Oryzomys russatus & 1 \\
\hline & Oxymycterus & Oxymycterus nasutus & 1 \\
\hline & & Oxymycterus dasytrichus & 2 \\
\hline & & Oxymycterus rufus & 1 \\
\hline & Pseudoryzomys & Pseudoryzomys simplex & 2 \\
\hline & Rhagomys & Rhagomys rufescens & 3 \\
\hline & Rhipidomys & Rhipidomys tribei & 1 \\
\hline & & Rhipidomys itoan & 1 \\
\hline & & Rhipidomys ipukensis & 2 \\
\hline & & Rhipidomys macrurus & 1 \\
\hline & & Rhipidomys tschudi & 1 \\
\hline & & Rhipidomys mastacalis & 1 \\
\hline & Scapteromys & Scapteromys sp & 1 \\
\hline & Sciurus & Sciurus aestuans & 1 \\
\hline & & Sciurus ingrami & 2 \\
\hline & & Sciurus spadiceus & 2 \\
\hline & Scolomys & Scolomys juruaense & 1 \\
\hline & & Scolomys ucayalensis & 1 \\
\hline & & Scolomys melanops & 1 \\
\hline & Sooretamys & Sooretamys angouya & 1 \\
\hline & Thalpomys & Thalpomys lasiotis & 4 \\
\hline & & Thalpomys nigrita & 3 \\
\hline & & Thalpomys cerradensis & 1 \\
\hline
\end{tabular}


conclusão

\begin{tabular}{|c|c|c|c|}
\hline Família & Gênero & Espécie & Quantidade de citações \\
\hline & Wiedomys & Wiedomys cerradensis & 1 \\
\hline & & Wiedomys pyrrhorhinus & 1 \\
\hline & & Wiedomys pyrrhorhinus & 1 \\
\hline \multirow[t]{7}{*}{ Ctenomyidae } & Ctenomys & & 3 \\
\hline & & Ctenomys flamarioni & 4 \\
\hline & & Ctenomys ibicuiensis & 1 \\
\hline & & Ctenomys lami & 4 \\
\hline & & Ctenomys minutus & 6 \\
\hline & & Ctenomys torquatus & 3 \\
\hline & & Ctenomys pearsoni & 1 \\
\hline \multirow[t]{4}{*}{ Dasyproctidae } & Dasyprocta & Dasyprocta azarae & 5 \\
\hline & & Dasyprocta fuliginosa & 1 \\
\hline & & Dasyprocta leporina & 1 \\
\hline & & Dasyprocta prymnolopha & 2 \\
\hline \multirow[t]{26}{*}{ Echimyidae } & Callistomys & Callistomys pictus & 1 \\
\hline & Clyomys & & 1 \\
\hline & & Clyomys bishopi & 2 \\
\hline & & Clyomys laticeps & 1 \\
\hline & Euryzygomatomys & Euryzygomatomys spinosus & 1 \\
\hline & Gyropus & & 1 \\
\hline & Makalata & Makalata didelphoides & 1 \\
\hline & Phyllomys & Phyllomys sulinus & 1 \\
\hline & & Phyllomys unicolor & 1 \\
\hline & Proechimys & & 1 \\
\hline & & Proechimys cayennensis & 1 \\
\hline & & Proechimys roberti & 1 \\
\hline & & Proechimys guyannensis & 1 \\
\hline & & Proechimys cuvieri & 2 \\
\hline & & Proechimys longicaudatus & 1 \\
\hline & Thrichomys & & 1 \\
\hline & & Thrichomys apereoides & 5 \\
\hline & & Thrichomys sp & 2 \\
\hline & & Thrichomys laurentius & 3 \\
\hline & & Thrichomys pachyurus & 1 \\
\hline & Trinomys & Trinomys yonenagae & 2 \\
\hline & & Trinomys albispinusminor & 2 \\
\hline & & Trinomys eliasi & 1 \\
\hline & & Trinomys iheringi & 1 \\
\hline & & Trinomys iheringi denigratus & 1 \\
\hline & & Trinomys gratiosus & 1 \\
\hline \multirow[t]{5}{*}{ Erethizontidae } & Chaetomys & Chaetomys subspinosus & 6 \\
\hline & Coendou & & 1 \\
\hline & & Coendou insidiosus & 1 \\
\hline & & Coendou spinosus & 1 \\
\hline & Sphiggurus & Sphiggurus villosus & 1 \\
\hline Muridae & Rattus & Rattus rattus & 1 \\
\hline
\end{tabular}


Figura 2 - Principais espécies citadas nos trabalhos publicados. A) Akodon montensis (extraído de: MYERS et al., 2013); B) Akodon cursor (extraído de: COUTINHO, 2013); C) Oligoryzomys nigripes (extraído de: SACRAMENTO, 2013).
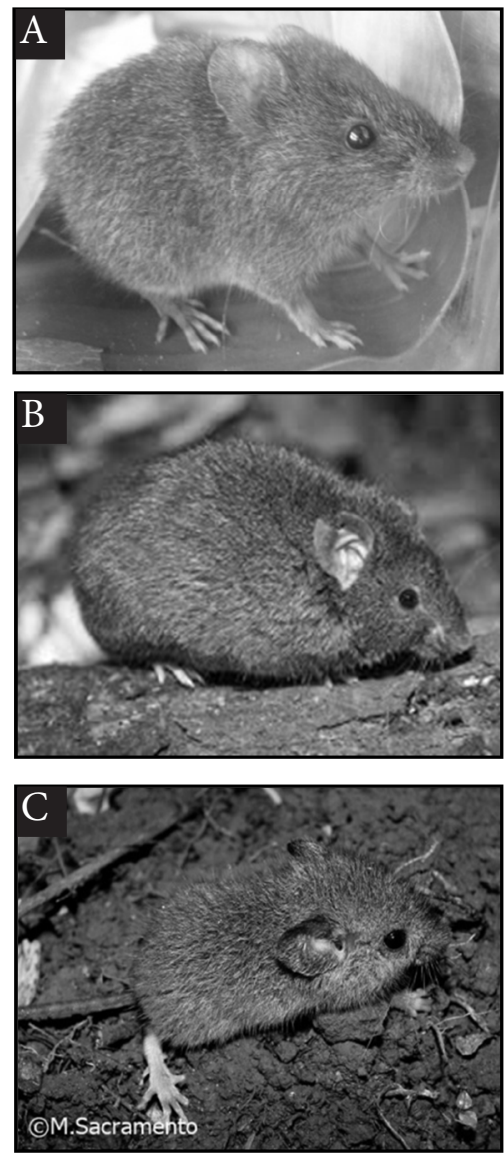

\section{Conclusões}

O levantamento da produção científica, no estado do Paraná e no Brasil, sobre os roedores, contribui para o conhecimento do estado da arte do grupo, identificando as principais características abordadas nos trabalhos. Reflete, também, o grande número de trabalhos realizados nas diversas áreas, principalmente em genética, em diferentes biomas brasileiros, especialmente na Mata Atlântica. Porém, os estudos com esse grupo devem ser ampliados, especialmente no Paraná, devido à escassez de trabalhos realizados em comparação com a grande diversidade existente.

Apesar do grande número de artigos sobre temas em ecologia, entende-se que é necessário ampliar os estudos da composição e inventário das espécies do grupo nos ambientes, por causa de sua importân- cia em fornecer subsídios no delineamento de áreas destinadas à conservação e detecção de áreas impactadas. Além disso, é necessário desenvolver mais pesquisas enfocando, também, as funções ecológicas desses roedores, para uma melhor compreensão do ecossistema como um todo.

Sugere-se, ainda, que as futuras pesquisas sobre o estudo quantitativo a respeito dos roedores sejam ampliadas, com a finalidade de reconhecer as características presentes nas produções científicas em outros períodos, bem como comparar com o estado da arte em outros estados brasileiros.

\section{Agradecimentos}

Ao Programa de Pós-Graduação em Biologia Comparada, da Universidade Estadual de Maringá, e à CAPES/ Fundação Araucária, pelo apoio financeiro concedido aos pós-graduandos bolsistas.

\section{Referências}

BERBERT, A. L. C. V. et al. Histological and serological evidence of experimental paracoccidioidomycosis in Calomys callosus (Rodentia: Cricetidae). International Journal of Experimental Pathology, v. 88, p. 55-62, 2007.

BONVICINO, C. R.; OLIVEIRA, J. A.; D'ANDREA, P. S. Guia dos Roedores do Brasil, com chaves para gêneros baseadas em caracteres externos. Rio de Janeiro: Centro Pan-Americano de Febre Aftosa - OPAS/OMS, 2008.

BRITO, D. Lack of adequate taxonomic knowledge may hinder endemic mammal conservation in the Brazilian Atlantic Forest. Biodiversity and Conservation, v. 13, p. 2135-2144, 2004.

COUTINHO, R. O Akodon cursor ou rato-da-mata. Disponível em <http://www.culturamix.com/animais/ roedores/o-akodon-cursor-ou-rato-da-mata $>$. Acesso em: 06 junho 2013.

EMMONS, L. H.; FEER, F. Neotropical rainforest mammals: a field guide. Chicago: University of Chicago, 1997.

GONÇALVES, A. Q.; PINTO, R. M.; DURETTE-DESSET, M. C. Parasitism of two zoonotic reservoirs Dasyprocta leporina and D. fuliginosa (Rodentia) from Amazonas, with Trichostrongylina nematodes (Heligmonellidae): description of a new genus and a new species. Memórias do Instituto Oswaldo Cruz, v. 102, n. 6, p. 763-768, 2007.

HERRERA, L. et al. Trypanosoma cruzi in a caviomorph rodent: parasitological and pathological features of the experimental infection of Trichomys apereoides (Rodentia, Echimyidae). Experimental Parasitology, v. 107, p. 78-88, 2004. 
LAINSON, R.; CARNEIRO, L. A.; SILVEIRA, F. T. Observations on Eimeria species of Dasyprocta leporina (Linnaeus, 1758) (Rodentia: Dasyproctidae) from the state of Para, North Brazil. Memórias do Instituto Oswaldo Cruz, v. 102, n. 4, p. 541-541, 2007.

MALDONADO JÚNIOR, A. et al. Helminth communities of Nectomys squamipes naturally infected by the exotic trematode Schistosoma mansoni in southeastern Brazil. Journal of Helminthology, v. 80, n. 4, p. 369-375, 2006.

METZGER, J. P. Conservation issues in the Brazilian Atlantic forest. Biological Conservation, v. 142, p. 11381140, 2009.

MINISTÉRIO DO MEIO AMBIENTE. Biodiversidade brasileira: Avaliação e identificação de áreas e ações prioritárias para a conservação, utilização sustentável e repartição dos benefícios da biodiversidade nos biomas brasileiros. Brasília: MMA, 2002.

MYERS, P. et al. The animal diversity web. Disponível em: <http://animaldiversity.ummz.umich.edu/accounts/ Akodon_montensis/>. Acesso em: 06 jun 2013.

NOWAK, R. M. Walker's Mammals of the World. Baltimore: The Johns Hopkins University Press, 1999.

PARDINI, R.; UMETSU, F. Pequenos mamíferos não-voadores da Reserva Florestal do Morro Grande: distribuição das espécies e da diversidade em uma área de Mata Atlântica. Biota Neotropica v. 6, n. 2, p. 3-22, 2006.

PATTERSON, B. D. On the continuing need for scientific collecting of mammals. Mastología Neotropical/ Journal of Neotropical Mammalogy, v. 9, n. 2, 253-262, 2002.

PINHEIRO, P. S.; HARTMANN, P. A.; GEISE, L. New record of Rhagomys rufescens (Thomas 1886) (Rodentia: Muridae: Sigmodontinae) in the Atlantic forest of southeastern Brazil. Zootaxa, v. 431, p. 1-11, 2004.

PREVEDELLO, J. A.; MENDONÇA, A. F.; VIEIRA, M. $\mathrm{V}$. Uso do espaço por pequenos mamíferos: uma análise dos estudos realizados no Brasil. Oecologia Brasiliensis, v. 12, n. 4, p. 610-625, 2008.

REIS, N. R. Mamíferos do Brasil. Londrina: Nelio R. dos Reis, 2011.

et al. Sobre os Mamíferos do Brasil. In: REIS, N.

R. Mamíferos do Brasil. Londrina: Nelio R. dos Reis, p. 23-29, 2011.

RIBEIRO, M. C. et al. The Brazilian Atlantic Forest: how much is left, and how is the remaining forest distributed? Implications for conservation. Biological Conservation, v. 142, p. 1141-1153, 2009.

SACRAMENTO, M. Oligoryzomys nigripes. Disponível em $<$ http://calphotos.berkeley.edu/cgi/img_query?enlar $\mathrm{ge}=0000+0000+1109+0302>$. Acesso em: 06 junho 2013.
SARAIVA, D. G. et al. Ticks (Acari: Ixodidae) associated with small terrestrial mammals in the state of Minas Gerais, southeastern Brazil. Experimental and Applied Acarology, v. 58, p. 159-166, 2012.

SMITH, M. F.; PATTON, J. L. Phylogenetic Relationships and the Radiation of Sigmodontine Rodents in South America: Evidence from Cytochrome b. Journal of Mammalian Evolution, 1999.

SPINAK, E. Indicadores cienciométricos. Ciência da Informação, v. 27, n. 2, p. 141-148, 1998.

STEIN, G. G. et al. Use of the anesthetic combination of tiletamine and zolazepam for immobilization of Ctenomys lami (Rodentia-Ctenomyidae) in southern Brazil. Acta Scientiae Veterinariae, v. 37, n. 3, p. 271-275, 2009.

TIEPOLO, L. M. Genética da conservação e filogenética, em espécies de mamíferos de pequeno porte da família Cricetidae (Rodentia) ocorrentes no litoral sul do estado do Paraná, através da citogenética (clássica e molecular). Disponível em: $<$ http://www.iap.pr.gov. $\mathrm{br} /$ arquivos/File/Pesquisa $\% 20 \mathrm{em} \% 20 \mathrm{UCs} /$ Projetos $\% 20$ de\%20Pesquisas\%20Autorizados\%20em\%202009/Liliani_ Marilia_Tiepolo.pdf>. Acesso em: 15 abril 2013.

ULLMANN, L. S. et al. Epidemiology of leptospirosis at Sorocaba Zoo, São Paulo state, Southeastern Brazil. Pesquisa Veterinária Brasileira, v.32, n.11, 2012.

WILSON, D. E.; REEDER, D. M. Mammal Species of the world: A taxonomic and geographic reference. Baltimore: Johns Hopkins University Press, 2005. 